\title{
Mejora en el comportamiento a la fatiga de compuestos nanoestructurados bidireccionales de fibra de vidrio y poliuretano bajo cargas uniaxiales $y$ altas deformaciones
}

\author{
Improved fatigue performance of nanostructured \\ bidirectional fiberglass-polyurethane composites \\ under uniaxial state and high deformations
}

Alberto Belardi ${ }^{1}$, Nicolás A. Oyarzabal ${ }^{1}$, Chiacchiarelli, Leonel Matías ${ }^{1,2}$

\author{
${ }^{1}$ Instituto Tecnológico de Buenos Aires (ITBA), Departamento de Ingeniería Mecánica, Av. E. Madero 399, CABA, Bue- \\ nos Aires, Argentina. \\ ${ }^{2}$ Instituto de Tecnología de Polímeros y Nanotecnología (ITPN), CONICET-FIUBA, Buenos Aires, Buenos Aires , Ar- \\ gentina. \\ e-mail: 1mchiacchiarelli@yahoo.com.ar
}

\section{RESUMEN}

Se fabricaron placas de material compuesto con refuerzo en fibra de vidrio bidireccional $[+/-45]_{4 S}$ y matriz poliuretano nanoestructurado usando la técnica de infusión asistida por vacío. Se utilizó nanosílice (NS) hidrofóbica con porcentajes del 1 y $2 \%$ en peso. A pesar de la NS, la permeabilidad de la preforma no aumentó significativamente, obteniéndose valores elevados de $\mathrm{V}_{\mathrm{f}}(52 \%)$ y una buena impregnación del refuerzo, comprobada mediante microscopía SEM. Los ensayos de tracción uniaxial cuasiestáticos presentaron una elevada deformación a rotura $\left(\mathrm{e}_{\max }>10 \%\right)$ y valores de resistencia última (RUTS) y módulo elástico (E) que disminuyeron en función del agregado de NS. Por el contrario, los ensayos de fatiga controlados por tensión indicaron que el agregado de NS hasta el 2\% permitió mejorar la performance en fatiga. En concreto, los parámetros a y b del modelo empírico lineal aumentaron su valor absoluto en un $77 \%$ y 92,7 \%, respectivamente. Esta mejora se corroboró a través de la evaluación del módulo elástico residual. Finalmente, los laminados presentaron una acumulación inelástica de deformación ("ratchetting"), aspecto que fue evaluado para dos frecuencias, $0.2 \mathrm{~Hz}$ y $2 \mathrm{~Hz}$.

Palabras-clave: poliuretano, fatiga, fibra de vidrio, nanocompuestos

\begin{abstract}
Plates of composite material of bidirectional fiberglass $[+/-45]_{4 \mathrm{~S}}$ and nanostructured polyurethane matrix were manufactured using the Vacuum Assisted Resin Infusion (VARI) technique. Hydrophobic nanosilica (NS) was used at 1 and 2 wt. $\%$ loading. Even though the use of NS, the permeability of the perform did not increase substantially, obtaining high $\mathrm{V}_{\mathrm{f}}$ $(52 \%)$ and a good impregnation, as visualized with SEM. Uniaxial tensile tests under quasi-static conditions presented high deformations to failure ( $\mathrm{e}_{\max }>10 \%$ ), tensile strengths (RUTS) and elastic moduli (E) which decreased as a function of increased NS loading. On the other hand, the stress-controlled fatigue testings revealed that the addition of NS up to $2 \mathrm{wt}$. $\%$ improved fatigue performance. Specifically, the $\mathrm{a}$ and $\mathrm{b}$ constants of the empirical lineal model presented an increase of $77 \%$ and $92.7 \%$, respectively. This improvement was corroborated through the measurement of the residual elastic modulus. Finally, the laminates presented an accumulation of inelastic deformation (ratcheting), whereas this phenomena was studied for two frequencies, $0.2 \mathrm{~Hz}$ and $2 \mathrm{~Hz}$.
\end{abstract}

Keywords: polyurethane, fatigue, fiberglass, nanocomposite 


\section{INTRODUCCIÓN}

El desarrollo de materiales compuestos de matriz polimérica con elevada resistencia a la fatiga es un aspecto clave para el impulso de las energías renovables. Para el caso de energía eólica, una turbina eólica tiene muchos componentes fabricados en materiales compuestos de matriz polimérica. Por ejemplo, la cáscara de la pala o aspa se fabrica con refuerzo en fibra de vidrio y matriz poliéster o epoxi. Teniendo en cuenta que una turbina eólica debe generar energía por períodos superiores a los 20 años, resulta importante reducir al máximo el daño generado en servicio. Esto se traduce en la posibilidad de certificar el material para tiempos mayores, extendiendo la vida útil del aspa y, por ende, la capacidad de producir energía a un menor costo.

Un aspecto clave para mejorar la resistencia a la fatiga de los materiales compuestos radica en evitar la formación y propagación de fisuras [1]. La presencia de poros en el material es un aspecto clave a tener en cuenta para aumentar la vida a la fatiga [2]. Los poros se comportan como defectos que disminuyen sensiblemente la resistencia local del material, aumentando el riesgo de propagación y falla catastrófica. En materiales compuestos de matriz polimérica, los poros se localizan en la matriz y la interfaz.

Actualmente, la mayoría de las matrices usadas para la fabricación de aspas eólicas se limitan a resinas poliéster insaturadas, decisión motivada principalmente por su bajo costo [3]. En el caso que se desee una mejor performance, se puede optar por sistemas epoxi o viniléster, sin embargo, el costo elevado de estos sistemas en general no permite que se realice esa transición. Otra opción es la utilización de matrices poliuretánicas [4]. Éstas poseen un excepcional comportamiento frente a la fatiga, propiedades mecánicas adecuadas y, además, su costo es menor que los sistemas epoxi. Sin embargo, uno de los grandes problemas de los sistemas poliuretánicos radica en su tendencia a la formación de poros durante el proceso de fabricación de piezas, particularmente cuando se utiliza el proceso de infusión asistida por vacío (IAV). En este caso, presiones inferiores a la atmosférica (vacío) son usadas para generar un gradiente de presión útil para la impregnación del refuerzo con la matriz y, además, para su posterior compactación. Sin embargo, este gradiente fomenta también la formación in-situ de burbujas de aire, dado que presiones inferiores a la atmosférica inducen el desgasado de la resina. Cabe destacar que esta formación de burbujas de aire ocurre principalmente en el frente de infusión [5]. Por el bajo espesor del frente de infusión, del orden de los milímetros, el avance del frente permite un desgasado in-situ de la resina, evitando la formación de poros y mejorando la resistencia a la fatiga del material compuesto. Sin embargo, este desgasado in-situ muchas veces no es suficiente, presentando laminados con elevada porosidad.

Es sabido que la utilización de nanopartículas es una excelente herramienta para la obtención de matrices nanoestructuradas (nanocompuestos) con mejores propiedades mecánicas, especialmente bajo cargas cíclicas [6]. Las nanopartículas poseen una inherente ventaja frente a los aditivos particulados micrométricos debido a que sólo es necesario formular la matriz con contenidos mucho menores al $10 \%$ en peso. Muchos trabajos han revelado mejorías para contenidos del orden del $1 \%$ en peso para el caso de nanosílice y del orden del $0.1 \%$ en peso para nanocelulosa. Un aspecto clave para la aplicación de estas matrices en el proceso de infusión asistida por vacío (IAV) radica en el hecho de que la nanopartícula no aumente demasiado su viscosidad. Un aumento significativo de la viscosidad implica una disminución significativa de la velocidad del frente de infusión, lo que implica que se tengan que diseñar zonas de infusión mucho más pequeñas, aumentando el costo del proceso. Sin embargo, varios estudios [2, 6-9] han determinado que este efecto puede llegar a ser despreciable. Otro aspecto relevante radica en el tiempo de gel de la matriz. Si las nanopartículas generan una disminución del tiempo de gel, esto afectará la productividad del proceso de IAV. Sin embargo, para la mayoría de los estudios cinéticos realizados con este propósito, se ha determinado que las nanopartículas provocan un aumento del tiempo de gel. Sólo para el caso de nanopartículas con funcionalidades específicas podrá ocurrir una diminución del tiempo de gel. Finalmente, varios estudios han demostrado que el agregado de nanopartículas permite lograr un aumento de la vida a la fatiga [2, 6-9]. De todas las nanopartículas estudiadas en la literatura, las que mayor potencial tienen de ser usadas en industria son las nanoarcillas y nanosílices. Éstas poseen bajo costo, no son tóxicas y su dispersión en la matriz se puede facilitar a través de su funcionalización. Sin embargo, las nanoarcillas son más difíciles de dispersar, dado que al poseer una estructura lamelar, es necesario que ocurra una exfoliación de sus capas lamelares para lograr un efecto importante en la matriz. Por otro lado, la nanosílice posee una geometría esférica y se dispersa en matrices termoestables en forma de tactoide. Como se ha determinado en estudios previos [10-12], no es estrictamente necesario que las nanopartículas esféricas estén perfectamente dispersas; si se pueden obtener tactoides no compactos se podrá observar un efecto importante en las propiedades de la matriz bajo estudio.

En este trabajo se propone la fabricación de materiales compuestos nanoestructurados usando una matriz de poliuretano y nanosílice como nanopartícula. Actualmente, la mayoría de las palas de una turbina eólica se fabrican usando principalmente matrices epoxi o poliéster convencionales. Como es comprobado por los resultados de este trabajo, el uso de matrices poliuretánicas nanoestructuradas permitirá el aumento de la perfomance a la fatiga. Para esto, se fabricaron placas de material compuesto con refuerzo en fibra de vidrio bidireccional $[+/-45]_{4 \mathrm{~S}}$ con matrices poliuretano y poliuretano nanoestructurado con nanosílice en porcentajes del 1\% y 2\% en peso. Se utilizó IAV para la fabricación de placas, obteniendo la permeabilidad a través del registro del avance del frente de infusión. Se evaluó el porcentaje en volumen de refuerzo 
usando microscopía electrónica de barrido (SEM), se analizaron las propiedades mecánicas bajo cargas uniaxiales para el caso de cargas aplicadas cuasiestáticamente y en fatiga. Se analizó el módulo elástico residual en función de la cantidad de ciclos para el caso de matrices poliuretano y poliuretano nanoestructuradas así como el efecto de la frecuencia en los ciclos a rotura.

\section{MATERIALES Y MÉTODOS}

\subsection{Materiales}

El poliuretano utilizado como matriz consistió en un poliol denominado RIMLINE SK 97011 y un isocianato de tipo polimérico derivado del diisocianato de difenilmetano (MDI) denominado comercialmente como SUPRASEC 9702. Ambos componentes fueron provistos por Huntsman Argentina.

Teniendo en cuenta que las dimensiones de la placa a fabricar están limitadas por el tiempo de gel de la matriz poliuretano (resina), se procedió a determinar el tiempo máximo de impregnación como el tiempo en el cual la matriz adquirió una viscosidad de $1000 \mathrm{cp}$. Teniendo en cuenta el perfil de viscosidad provisto por Huntsman, el máximo tiempo de impregnación fue de 15 minutos a $24^{\circ} \mathrm{C}$.

Antes de la preparación de las placas por el método de infusión, se procedió a desgasar ambos componentes por separado a $30 \mathrm{mbar}$, a una temperatura de $40^{\circ} \mathrm{C}$ y agitando a $3000 \mathrm{rpm}$ por un tiempo de 40 minutos. Este procedimiento se realizó en un dispersor de alta velocidad diseñado y fabricado por nuestro grupo de investigación.

Como refuerzo se utilizó un tejido bidireccional $\left(+/-45^{\circ}\right)$ de fibra de vidrio con gramaje de 300 g.m $\mathrm{m}^{-2}$ obtenido de CPIC Argentina. El tejido poseía un sizing de aminosilano adecuado para su uso con matrices poliuretánicas. La nanopartícula utilizada fue una nanosílice (NS) denominada comercialmente AEROSIL R8200, provista por Evonik Argentina. La dispersión de la NS en la matriz poliuretánica se realizó incorporando la NS en el poliol en concentraciones del 1\% y 2\% en peso. El proceso de dispersión se realizó con el equipo mencionado anteriormente usando un agitador tipo Cowles a una velocidad de rotación de $10.000 \mathrm{rpm}$, vacío de $30 \mathrm{mbar}$ y temperatura $40^{\circ} \mathrm{C}$ por un tiempo total de $10^{\prime}$. Este procedimiento permitió obtener una dispersión nanométrica de la NS en la matriz poliuretánica, como se ha comprobado en trabajos anteriores de Chiacchiarelli et al. [10,12].

\subsection{Proceso de fabricación de placas por infusión asistida por vacío (IAV)}

Se fabricaron placas de $500 \mathrm{~mm}$ de largo y $290 \mathrm{~mm}$ de ancho. Para cada tipo de matriz, PUR, PUR 1\% NS y PUR 2\% NS, se fabricaron dos placas.

Se utilizó la infusión asistida por vacío (IAV), del inglés "Vacuum Assisted Resin Infusion" para fabricar placas para su posterior ensayo. En la Figura 1 se puede observar la disposición de los elementos usados para la fabricación de placas de material compuesto nanoestructurado, siguiendo los lineamientos habitualmente usados en el estado del arte [13]. En detalle, se nota que el sistema de infusión consistió en un embudo de inyección de resina puesto en serie con una válvula esférica. La salida de la válvula estaba conectada directamente a la entrada de la zona de infusión. Para lograr un frente plano de infusión, los primeros $10 \mathrm{~cm}$ del frente de infusión fueron cubiertos con dos capas de trama de flujo, de forma tal de aumentar la velocidad de flujo de la resina, logrando un flujo planar en pocos segundos. La salida estaba conectada también a otra válvula esférica, la cual estaba conectada a una trampa de vacío utilizada para evitar contaminación de la bomba de vacío y, a su vez, a la bomba de vacío (Dosivac DVR3A 140 l.min ${ }^{-1}$ ). Como puede notarse en la Figura 1, la delimitación de la placa se selló usando nódulo de tipo butílico doble. Es decir, dejando una zona intermedia que permita morigerar hipotéticas pérdidas de integridad del sistema. Como molde, se utilizó una placa plana de aluminio, la cual fue pulida y limpiada con acetona previamente a la introducción de la preforma. 


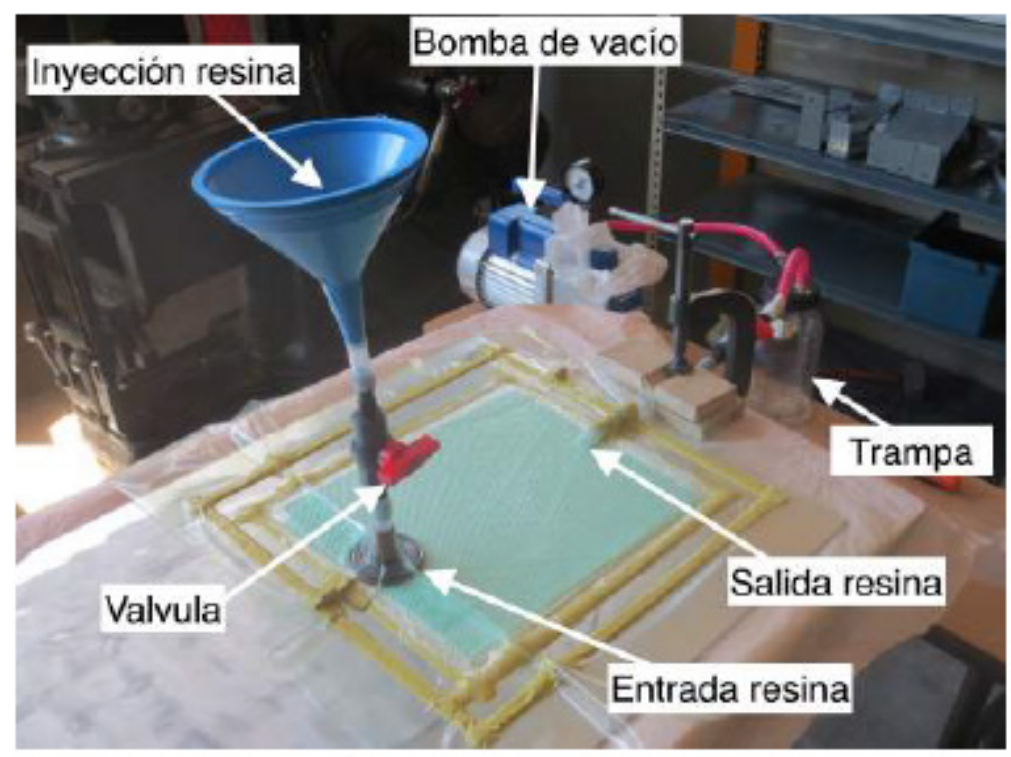

Figura 1: Disposición de elementos para la fabricación de placas usando IAV

Para fabricar una placa, se procedió a realizar los siguientes pasos. Primero se limpió el molde de aluminio con acetona y se dejó evaporar completamente. Se delimitó la zona de infusión con cinta de papel y se procedió a aplicar desmoldante (Tarnopol N40) en cuatro capas. Posteriormente, se despegó la cinta de papel y, en su lugar, se colocó la cinta de sellado (Airtech AT400), dejando el film de polietileno de forma tal de no contaminar el adhesivo de la cinta de sellado. Después se procedió a depositar los tejidos de fibra de vidrio bidireccional, pesándolos antes de presentarlos en el molde. Se colocaron 4 tejidos bidireccionales, obteniendo un gramaje superficial de 1200 g.m-2 . A continuación se depositó el peel-ply, utilizado para evitar que el laminado quede adherido a la bolsa de vacío. Arriba de esto se agregó la trama de flujo, la cual fue utilizada para acelerar el flujo en la dirección longitudinal de la placa. Es importante destacar que se tuvo que adecuar la permeabilidad de la trama de flujo de forma tal de evitar impregnaciones parciales en el espesor del laminado. Finalmente, se procedió a colocar la bolsa de vacío evitando cualquier solape y haciendo los pliegues necesarios para evitar discontinuidades. El próximo paso consistió en la prueba de integridad del sistema de vacío. Se procedió a encender la bomba de vacío abriendo las válvulas esféricas de la entrada y salida del panel. Se procedió a cerrar ambas válvulas y se comprobó que el sistema no tenía pérdidas de vacío mayores a 4 mbar por un rango de tiempo de al menos 40 minutos. Después de comprobar esto, se procedió a encender la bomba de vacío abriendo sólo la válvula esférica de la salida. Se procedió a mezclar los componentes de la matriz de poliuretano y se la introdujo en el embudo de inyección. Se abrió lentamente la válvula esférica de entrada y se procedió a cronometrar el frente de infusión a medida que impregnaba la placa. Cuando el frente de impregnación llegó a la salida de la placa, se cronometraron otros cinco minutos para favorecer la impregnación en el espesor del laminado. Finalmente, se procedió a cerrar la válvula de entrada y de salida, dejando la placa lista para su curado a temperatura ambiente. Después de 24 hs, se desmoldó la placa y se procedió a realizar una post-cura en un horno de convección forzada a $100^{\circ} \mathrm{C}$ por 1 hora.

\subsection{Ensayos mecánicos cuasiestáticos y de fatiga}

Las probetas utilizadas para los ensayos cuasiestáticos y de fatiga fueron mecanizadas usando una fresadora CNC (Haas TM-1) con una fresa integral de metal duro de 9 filos, $6 \mathrm{~mm}$ de diámetro, especial para materiales compuestos (código EPNF060S15-3L/9C06F57 IC02 Iscar Tools Argentina S.A.). Las dimensiones de las probetas fueron de $230 \mathrm{~mm}$ de largo por $20 \mathrm{~mm}$ de ancho. Posteriormente, se procedió al pegado de tabs (40 $\mathrm{mm}$ de largo y $20 \mathrm{~mm}$ de ancho y $3 \mathrm{~mm}$ de espesor) en los extremos de sujeción de la probeta siguiendo las recomendaciones de la norma ASTM D3039-08.

Los ensayos cuasiestáticos fueron realizados por triplicado en una máquina de ensayos universal Instron 3382 de $100 \mathrm{kN}$ de capacidad, siguiendo los lineamientos de la normativa ASTMD3039-08. La misma cuenta con mordazas mecánicas de cuña con caras serradas, celda de carga de $100 \mathrm{kN}$ y extensómetro. Los ensayos fueron controlados por deformación, imponiendo un desplazamiento del cabezal de $2 \mathrm{~mm} \cdot \mathrm{min}^{-1}$. Mediante los mismos se obtuvo la resistencia máxima a la tracción (RUTS) y el módulo de Young del laminado (E). La medición del módulo elástico (E) se realizó utilizando un extensómetro axial estático "clip-on" (Instron) de longitud calibrada de $50 \mathrm{~mm}$ y recorrido $+/-5 \mathrm{~mm}$ usando los lineamientos de las normativas ASTM E83 e ISO 9513. 
Los ensayos de fatiga uniaxial en tensión-tensión fueron realizados a temperatura ambiente con un $\mathrm{R}=\mathrm{S}_{\min } \cdot \mathrm{S}_{\max }{ }^{-1}=$ 0.1 y una frecuencia de $2 \mathrm{~Hz}$ en una máquina servohidráulica Instron 8801 de $100 \mathrm{kN}$ de capacidad dotada de mordazas hidráulicas. Para los mismos se siguieron los lineamientos de la norma ASTM D3479. La selección de la tensión máxima del ensayo se realizó en función de la resistencia última (RUTS) de cada material. En otras palabras, los ensayos de fatiga se realizaron a tensiones expresadas en fracciones porcentuales de la RUTS (\%RUTS). Cada tipo de probeta se ensayó por triplicado a valores de \%RUTS del 20, 40, 50, 60 y $80 \%$. Por ejemplo, para el caso del [+/-45] $]_{45}$-PUR (Tabla 1), la RUTS medida fue de $130 \mathrm{MPa}$. Por ende, los ensayos a fatiga se realizaron con un $\mathrm{s}_{\text {max }}$ de $104 \mathrm{MPa}, 78 \mathrm{MPa}, 65 \mathrm{MPa}, 52 \mathrm{MPa}$ y $26 \mathrm{MPa}$, respectivamente. Usando un $\mathrm{R}=0.1$ se podrá deducir la respectiva $\mathrm{s}_{\min } \mathrm{y} \mathrm{s}_{\text {med }}$.

La determinación del módulo elástico residual $\left(\mathrm{E}_{\mathrm{r}}\right)$ se realizó combinando los ensayos de fatiga y los cuasiestáticos. En función de la cantidad total de ciclos a rotura del material bajo estudio, se procedió entonces a realizar mediciones del módulo elástico residual $\left(\mathrm{E}_{\mathrm{r}}\right)$ acumulando 200 ciclos entre cada medición. En otras palabras, un ensayo de $\mathrm{E}_{\mathrm{r}}$ consistió primero en ciclar la probeta en la máquina de fatiga por 200 ciclos, para después retirarla e introducirla en la máquina de tracción convencional para hacer mediciones de módulo elástico cuasiestático. Esto se repitió hasta llegar a los ciclos a rotura de cada probeta bajo análisis. Los ensayos se realizaron por triplicado y se muestran aquellos representativos.

\subsection{Análisis de la microestructura usando SEM}

El análisis de la microestructura de las probetas se realizó mediante micrografías SEM de muestras obtenidas en zonas representativas, siguiendo los lineamientos de trabajos anteriores del autor [14, 15]. La preparación de ésta consistió en su inclusión en acrílico y su posterior lijado y pulido teniendo en cuenta que para poder observar adecuadamente el refuerzo, cada probeta se rotó en $45^{\circ}$. Esto permitió observar las zonas de matriz y refuerzo en orientaciones de $0^{\circ}$ y $90^{\circ}$. La obtención de la fracción en volumen de refuerzo $\left(\mathrm{V}_{\mathrm{f}}\right)$ se realizó utilizando el método de las áreas usando la siguiente ecuación:

$$
V_{f}=\frac{A_{f}}{A}
$$

Aquí, $A_{f}$ y $A$ son, respectivamente, el área total del refuerzo y el área total de la región seleccionada.

La metodología implementada para la determinación cuantitativa de la $\mathrm{V}_{\mathrm{f}}$ consistió en el análisis estadístico de imágenes mediante el software ImageJ. Para obtener el valor $\mathrm{V}_{\mathrm{f}}$ se tomaron 20 imágenes SEM para cada tipo de material, cubriendo un área total de $2 \cdot 10^{-5} \mathrm{~mm}^{2}$.

\subsection{Modelo de flujo}

Teniendo en cuenta las características del proceso utilizado en este trabajo, el flujo de matriz poliuretánica se puede considerar equivalente al de un fluido incompresible a través de un medio poroso. Bajo ésta hipótesis, se podrá describir el problema aplicando la ecuación de Darcy y la conservación de masa [16]. Si se agrega la condición de un flujo planar unidimensional, la resolución del problema es analítica y la relación entre la distancia $x_{f}$ de avance del frente de infusión y el tiempo $t$ es la siguiente:

$$
x_{f}^{2}=\frac{2 K P_{a t m}}{\mu} t
$$

Aquí $K$ es la permeabilidad del medio poroso, $P_{\text {atm }}$ es la presión atmosférica y $\mu$ la viscosidad cinemática.

En la fase de diseño del tamaño de la placa, se procedió a calcular el máximo avance del frente de infusión teniendo en cuenta el tiempo al cual la viscosidad de la resina aumentó por arriba de $1000 \mathrm{cp}$ (15 minutos).

\section{RESULTADOS}

\subsection{Determinación de permeabilidad de la preforma}

La posición del frente de infusión $\left(\mathrm{x}_{\mathrm{f}}\right)$, expresada al cuadrado, en función del tiempo para las placas [+/-45] $]_{4 \mathrm{~S}}$ fabricadas con la matriz PUR, PUR 1\% NS y PUR 2\% NS se puede observar en la Figura 2. En todos los casos, la relación entre $\mathrm{x}_{\mathrm{f}}^{2} \mathrm{y}$ el tiempo fue lineal, con una disminución de la pendiente en función del agregado de NS. Teniendo en cuenta que una $\mathrm{P}_{\text {atm }}$ de $101325 \mathrm{~Pa}$, se pudo calcular el valor de K/ $\mu$ a través de una regresión lineal. Para el caso del PUR se obtuvo un valor de $8.10 .10^{-9} \mathrm{~m}^{2} . \mathrm{cps}^{-1}$ siendo el coeficiente de la correlación lineal 0.995 . Si se tiene en cuenta un valor promedio de $\mu$ de $350 \mathrm{cp}$ se puede deducir que la permeabilidad tuvo un valor de $2.8 .10^{-9} \mathrm{~m}^{2}$. Para el caso del PUR $1 \%$ NS y el $2 \%$ NS, las pendientes tuvieron los valores de $6.71 .10^{-9} \mathrm{~m}^{2} . \mathrm{cp}^{-1}$ y $4.29 .10^{-9} \mathrm{~m}^{2} . \mathrm{cp}^{-1}$, respectivamente. 


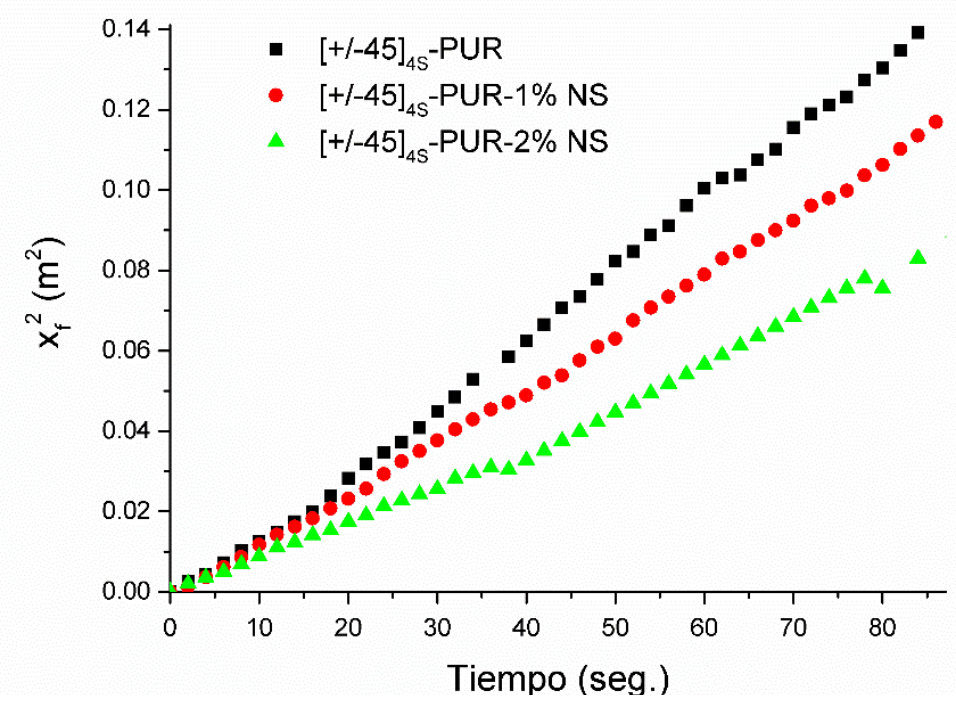

Figura 2: Posición del frente de infusión, expresado al cuadrado, en función del tiempo para las placas [+/-45 $]_{4 \mathrm{~S}}-\mathrm{PUR}$, $[+/-45]_{4 \mathrm{~S}}$-PUR $1 \% \mathrm{NS}$ y $[+/-45]_{4 \mathrm{~S}}$-PUR $2 \% \mathrm{NS}$.

\subsection{Determinación de la fracción en peso de refuerzo y matriz}

La determinación de $\mathrm{V}_{\mathrm{f}}$ se realizó en forma estadística a través de la observación de la superficie usando micrografías SEM. En la Figura 3 se pueden observar micrografías representativas de los materiales estudiados.

Para el caso del material compuesto [+/-45] $]_{4 \mathrm{~S}}-\mathrm{PUR}$, el valor promedio de $\mathrm{V}_{\mathrm{f}}$ fue de $52.3+/-0.1 \%$. Para el caso de $[+/-45]_{4 \mathrm{~S}}$-PUR-1\% NS y el $[+/-45]_{4 \mathrm{~S}}$-PUR-2\% NS, fueron $54.3+/-0.2 \%$ y $55.0+/-0.2 \%$, respectivamente.

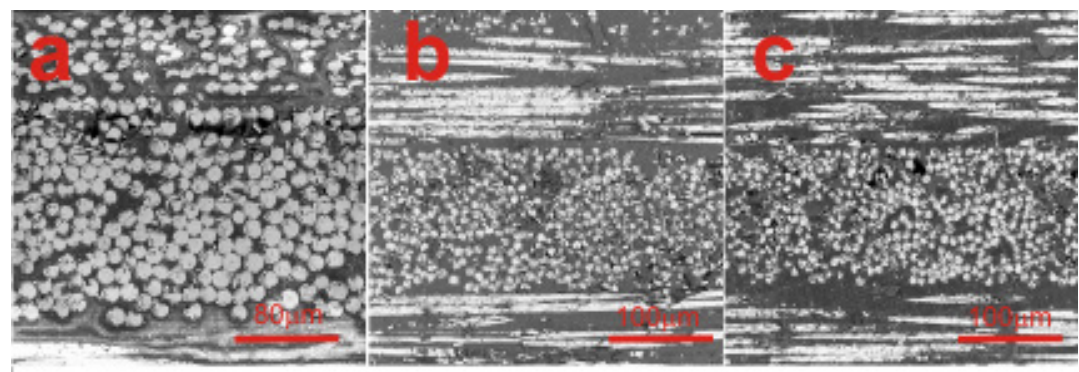

Figura 3: Micrografías SEM de la superficie de a) $[+/-45]_{4 \mathrm{~S}}-\mathrm{PUR}$, b) $[+/-45]_{4 \mathrm{~S}}-\mathrm{PUR} 1 \% \mathrm{NS}$ y c) $[+/-45]_{4 \mathrm{~S}}-\mathrm{PUR} 2 \%$ NS.

\subsection{Ensayos de tracción cuasiestáticos}

Las curvas de tensión nominal versus deformación nominal de las probetas ensayadas a tracción para los materiales compuestos estudiados en este trabajo se pueden observar en la Figura 4. Los valores de resistencia última (RUTS), módulo elástico (E) y la deformación a rotura $\left(\mathrm{e}_{\max }\right)$ en función del contenido de NS están listados en la Tabla 1 para todos los laminados fabricados con una secuencia $[+/-45]_{4 \mathrm{~S}}$.Para el caso de la RUTS, se observó que el agregado de NS provocó una disminución del valor de RUTS de hasta el 33\% para el caso de $[+/-45]_{45}$-PUR 2\% NS. La dispersión del valor medio de la RUTS aumentó con el agregado de NS, indicando que las nanopartículas generaron un material con propiedades más heterogéneas. Con respecto al módulo elástico (E), el agregado de NS generó una disminución de hasta el 30\% del valor medio para el caso del $[+/-45]_{4 \mathrm{~S}}$-PUR $2 \%$ NS. Finalmente, la deformación a rotura también disminuyó en función del agregado de NS, con una disminución del 16\% para el caso del $[+/-45]_{4 S}$-PUR $2 \%$ NS. 


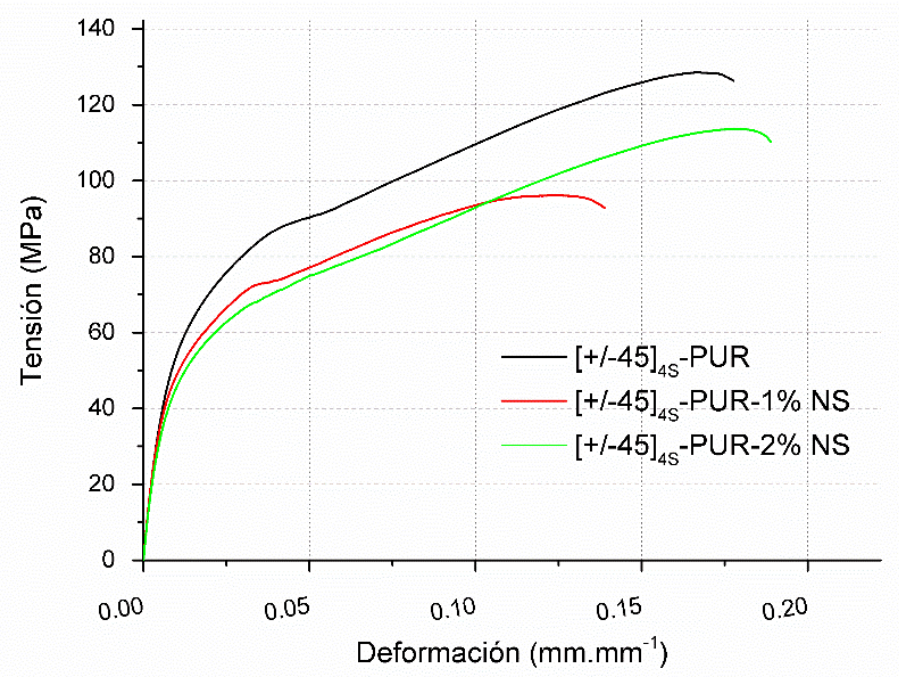

Figura 4: Tensión versus deformación para ensayos cuasiestáticos. Ver en la Tabla 1 el análisis estadístico.

Tabla 1: Propiedades mecánicas de las probetas ensayas en condiciones cuasiestáticas

\begin{tabular}{c|c|c|c}
\hline PROBETA & $\begin{array}{c}\text { RESISTENCIA ÚLTIMA } \\
\text { (RUTS, MPA) }\end{array}$ & $\begin{array}{c}\text { MÓDULO ELÁSTICO } \\
\text { (E, MPA) }\end{array}$ & $\begin{array}{c}\text { DEFORMACIÓN A } \\
\text { ROTURA (E }\end{array}$ MAx $^{\text {MM }}{ }^{-1}$ ) \\
\hline$[+/-45]_{45}$-PUR & $1.30 .10^{2}+/-1.40 .10^{0}$ & $8.55 .10^{3}+/-8.30 .10^{2}$ & $17.9 .10^{-2}+/-1.40 .10^{-3}$ \\
\hline$[+/-45]_{45}$-PUR 1 \% NS & $1.05 .10^{2}+/-12.8 .10^{0}$ & $7.53 .10^{3}+/-1.69 .10^{3}$ & $1.80 .10^{-2}+/-4.30 .10^{-3}$ \\
\cline { 4 - 4 } [+/-45 $]_{45}$-PUR 2 \% NS & $8.70 .10^{2}+/-27.1 .10^{0}$ & $5.96 .10^{3}+/-8.71 .10^{2}$ & $1.50 .10^{-2}+/-4.6 .10^{-3}$ \\
\hline
\end{tabular}

\subsection{Ensayos de fatiga}

Los resultados de los ensayos de fatiga de los materiales compuestos con refuerzo [+/-45] $]_{4 \mathrm{~S}}$ y matriz PUR, PUR $1 \%$ NS y PUR 2\% NS se muestran en la Figura 5. El gráfico muestra el \%RUTS (escala logarítmica) en función de los ciclos a rotura (Nf, en escala logarítmica).

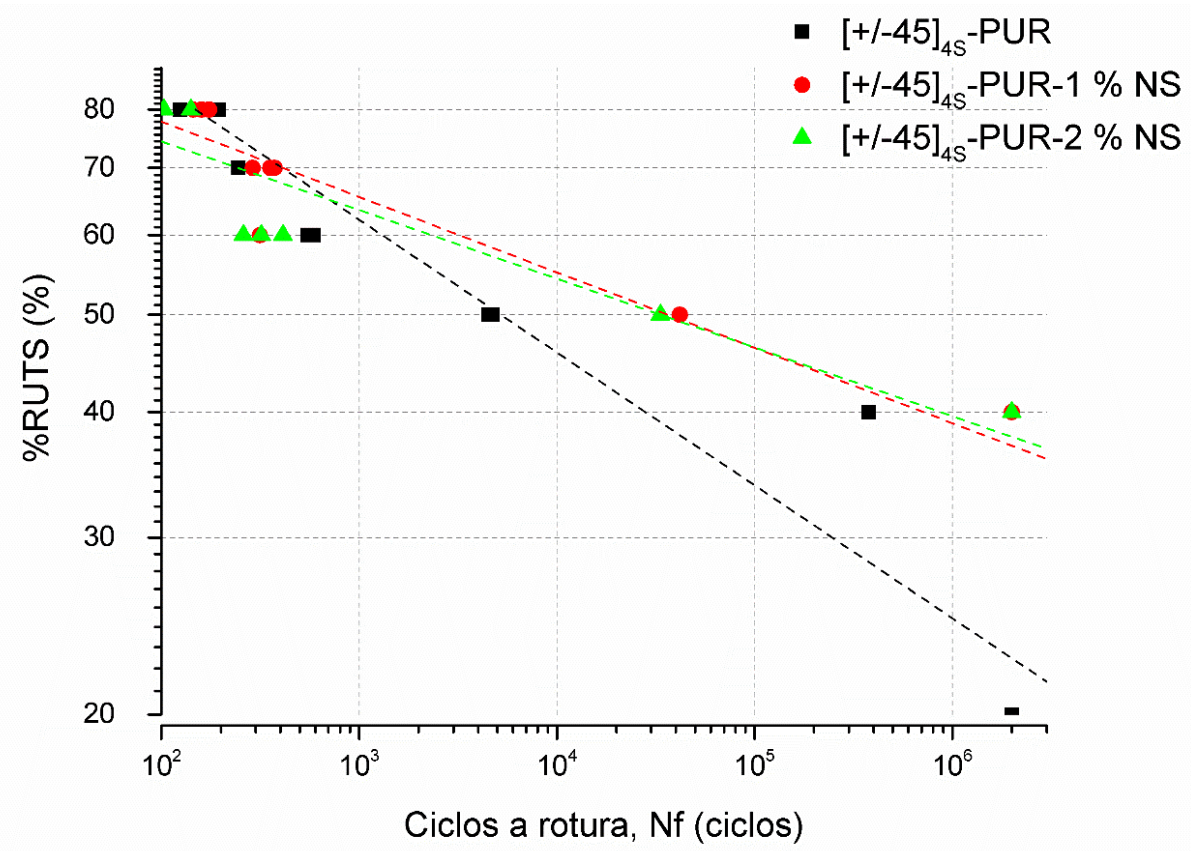

Figura 5: \%RUTS en función del número de ciclos a rotura para las probetas $[+/-45]_{4 \mathrm{~S}}-\mathrm{PUR},[+/-45]_{4 \mathrm{~S}}-\mathrm{PUR} 1 \%$ NS y $[+/-45]_{4 \mathrm{~S}}$-PUR $2 \% \mathrm{NS}$. 
Para modelar el comportamiento a la fatiga, se utilizó un modelo empírico de dos constantes basado en la siguiente ecuación:

$$
\log (S r)=a \log (\% R U T S)+b
$$

Aquí a y b representan constantes empíricas que fueron determinadas mediante una regresión lineal de los resultados obtenidos y que se encuentran listados en la Tabla 2.

Como puede deducirse de la Tabla 2, el agregado de NS al $2 \%$ aumentó en un 77\% el valor absoluto de la constante a del modelo indicado en la ecuación 2. Asimismo, para el caso del parámetro b, su valor absoluto aumentó un $92.7 \%$ para el caso de NS al 2\%. Las predicciones del modelo han sido agregadas a la Figura 5 en forma de líneas partidas.

Tabla 2: Valores de las constantes del modelo de fatiga empírico de dos parámetros.

\begin{tabular}{c|c|c|c}
\hline PROBETA & CONSTANTE A & CONSTANTE B & $\begin{array}{c}\text { COEFICIENTE DE } \\
\text { CORRELACIÓN }\end{array}$ \\
\hline$[+/-45]_{4 \mathrm{~S}}$-PUR & $1.66 .10^{1}+/-3.00 .10^{-1}$ & $-7.59 .10^{1}+/-0.2$ & 0.98 \\
\hline$[+/-45]_{4 \mathrm{~S}}$-PUR 1 \% NS & $2.72 .10^{1}+/-6.00 .10^{-1}$ & $-1.33 .10^{1}+/-3.00 .10^{-1}$ & $9.50 .10^{-1}$ \\
\cline { 3 - 4 }$[+/-45]_{4 \mathrm{~S}}$-PUR 2 \% NS & $2.94 .10^{1}+/-9.00 .10^{-1}$ & $-1.46 .10^{1}+/-5.00 .10^{-1}$ & $9.00 .10^{-1}$ \\
\hline
\end{tabular}

\subsection{Módulo elástico residual}

El módulo elástico residual (Er) en función de los ciclos de fatiga para probetas [+/-45] $]_{4 \mathrm{~S}}-\mathrm{PUR},[+/-45]_{4 \mathrm{~S}}-\mathrm{PUR} 1 \%$ NS y $[+/-45]_{4 \mathrm{~S}}$-PUR $2 \%$ NS ensayadas con un \%RUTS del $60 \%$ se muestra en la Figura 6. Como puede notarse, en todos los casos el Er disminuyó a medida que aumentaron los ciclos de fatiga. Asimismo, se observa que para el caso del [+/-45] $]_{45}-$ PUR, la disminución de $\mathrm{E}$ se acelera drásticamente a partir de aproximadamente 1400 ciclos, llegando muy rápidamente a la rotura final de la probeta. Para el caso de las probetas con 1 y $2 \%$ de NS, se observó un comportamiento similar, excepto que el valor a partir del cual el E disminuye drásticamente aumentó, indicando una mejora en el comportamiento a la fatiga de los laminados nanoestructurados. Es importante destacar que, a pesar de haber realizado los experimentos por triplicado, dada la variación estadística de las propiedades mecánicas de los materiales bajo estudio, los datos representados en la Figura 6 van a sufrir modificaciones acorde a lo esperado estadísticamente (Tabla 1).

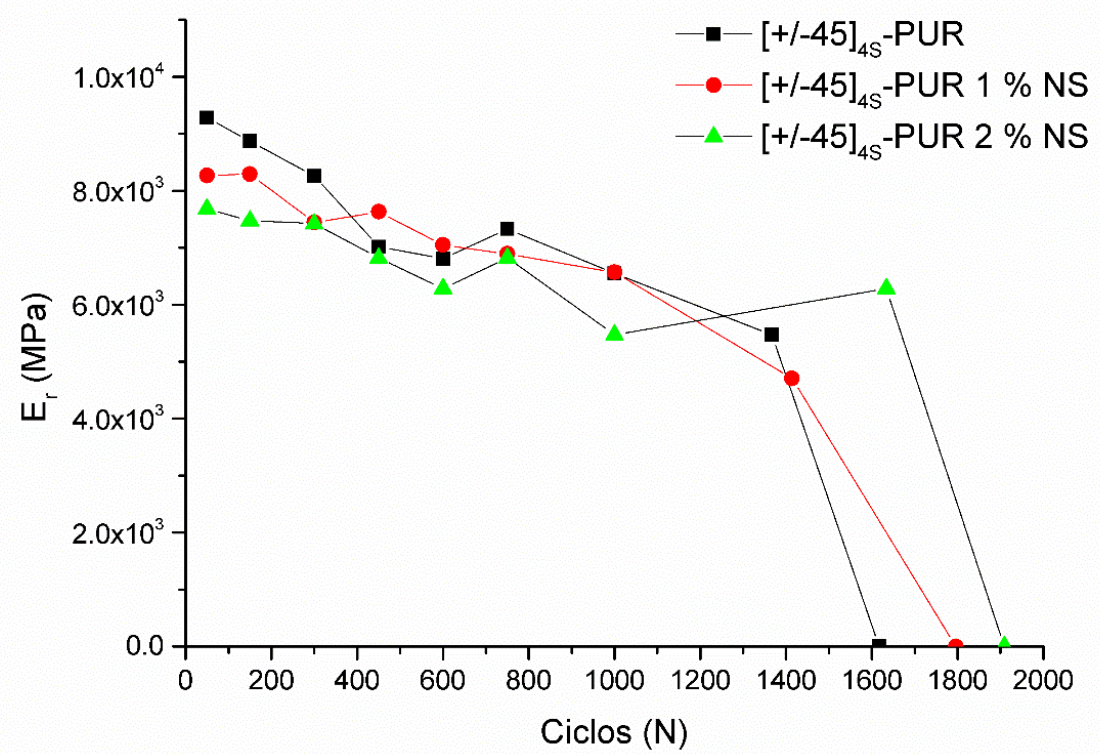

Figura 6: Módulo elástico residual (Er) en función de los ciclos de fatiga para las probetas $[+/-45]_{4 \mathrm{~S}}-\mathrm{PUR},[+/-45]_{4 \mathrm{~S}}-\mathrm{PUR}$ $1 \%$ NS y $[+/-45]_{4 S}$-PUR $2 \%$ NS ensayadas para un \%RUTS del $60 \%$.

\subsection{Análisis del efecto de la frecuencia}

El análisis del efecto de la frecuencia para probetas fabricadas con [+/-45] $]_{45}$-PUR y bajo una tensión de $60 \%$ RUTS a frecuencias de $2 \mathrm{~Hz}$ y $0.2 \mathrm{~Hz}$ se muestra en la Figura 7 . En el gráfico se puede observar la posición del cabezal en función de la 
cantidad de ciclos. Como podrá notarse, existen líneas horizontales que corresponden a la posición del cabezal durante un ciclo de fatiga (se ha modificado la figura para que pueda ser distinguida visualmente), mientras que las líneas en la dirección vertical son asociadas a la acumulación de deformación inelástica que provoca aumentos significativos de la posición del cabezal a medida que se acumulan ciclos.

Para una frecuencia específica, se puede notar que en ambas probetas existe un aumento considerable de la posición del cabezal a medida que crecen la cantidad de ciclos aplicados en una probeta. Asimismo, el aumento de la posición del cabezal dependió fuertemente de la frecuencia. Para menores frecuencias, se necesitó realizar una mayor cantidad de ciclos para llegar a una posición de cabezal específica. Por ejemplo, la probeta que fue ensayada a $2 \mathrm{~Hz}$ logró llegar a una posición de cabezal de $10 \mathrm{~mm}$ después de haber transcurrido 25 ciclos. Sin embargo, para el caso de la probeta ensayada a $0.2 \mathrm{~Hz}$, debieron transcurrir 100 ciclos para llegar a dicha posición.

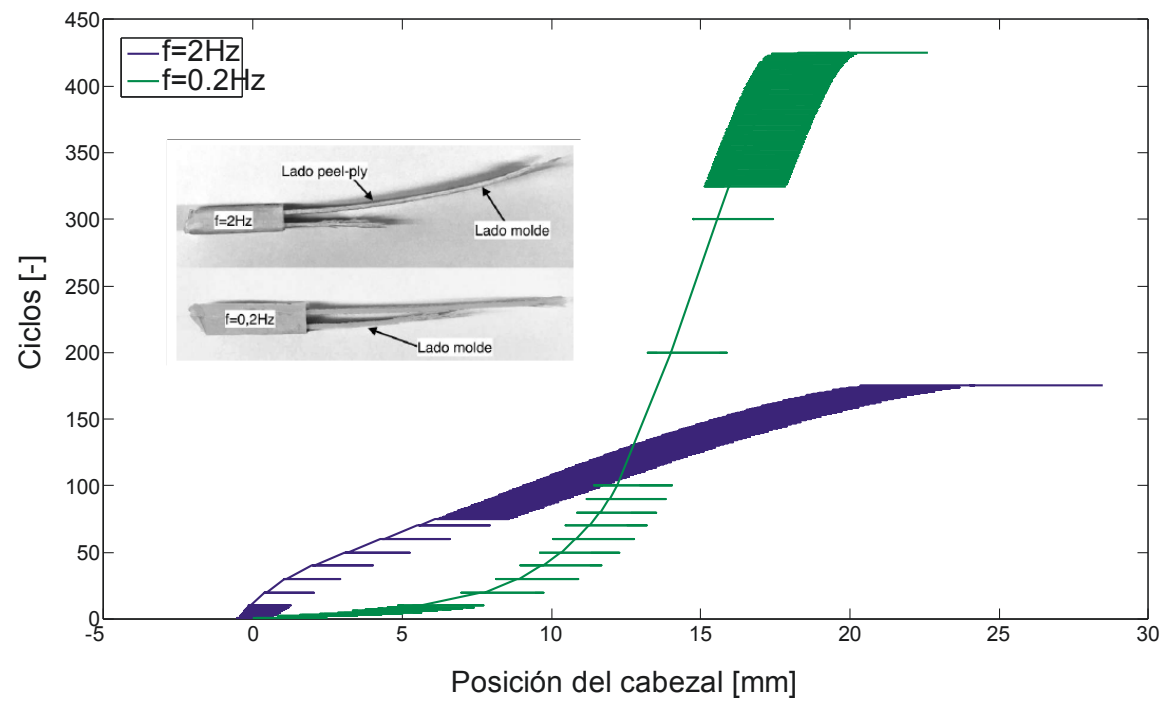

Figura 7: Ciclos en función de la posición del cabezal para una probeta $[+/-45]_{4 \mathrm{~S}}$-PUR ensayada a $0.2 \mathrm{~Hz}$ y $2 \mathrm{~Hz}$. La figura interna muestra las deformaciones residuales de cada probeta.

\section{DISCUSIÓN}

Los valores de las mediciones de permeabilidad de la preforma presentadas en la sección 3.1 son similares a los habitualmente encontrados en literatura $[17,18]$. La disminución de la pendiente de la Figura 2 se puede explicar teniendo en cuenta que el agregado de NS en la formulación causó un aumento de la viscosidad del poliuretano utilizado en el proceso de infusión asistida por vacío. Teniendo en cuenta los cálculos de permeabilidad obtenidos para la placa $[+/-45]_{4 \mathrm{~S}}-\mathrm{PUR}$, se puede deducir que la viscosidad aumentó un $19.2 \%$ para el $[+/-45]_{45}$-PUR $1 \%$ NS y un $86 \%$ para el $[+/-45]_{45}-$ PUR $2 \%$ NS. Estos resultados evidencian una de las desventajas del uso de nanopartículas, es decir, el aumento inherente de la viscosidad y la consecuente disminución de la velocidad del frente de infusión. Sin embargo, este efecto puede tenerse en cuenta durante el proceso de infusión limitando la máxima distancia de la zona de infusión y teniendo en cuenta que para el caso de una matriz poliuretánica nanoestructurada con 1 y $2 \%$ de NS, los tiempos de infusión aumentan en un $25 \%$ y un $62 \%$, respectivamente. Es importante tener en cuenta que el agregado de NS también aumenta el tiempo de gel de la matriz [19-21], por ende, podría ser factible utilizar la misma matriz sin necesidad de hacer cambios importantes en las zonas de infusión.

Las mediciones obtenidas de $\mathrm{V}_{\mathrm{f}}$ indican que el método de infusión permitió obtener placas con un elevado contenido de refuerzo, similar a lo habitualmente encontrado en literatura [22]. Asimismo, de la Figura 3 se puede deducir que en todos los casos estudiados en este trabajo, la impregnación micrométrica y macrométrica fueron adecuadas [23]. La impregnación micrométrica está controlada fundamentalmente por el efecto capilar generado por la superposición de los filamentos. A su vez, este fenómeno es fuertemente influenciado por la tensión superficial y por el agente de acople (del inglés "sizing") del refuerzo. Como puede notarse en la Figura 3, la zona micrométrica está correctamente impregnada. Por otro lado, la zona macrométrica está asociada a las zonas delimitadas por las agrupaciones de filamentos de refuerzo (mechas). Esta zona también puede notarse en la Figura 3 y, a su vez, se puede deducir que ha ocurrido una correcta impregnación macrométrica. Otro resultado que debe tenerse en cuenta tiene que ver con el hecho de que las placas fabricadas por PUR $2 \%$ NS presentaron un $\mathrm{V}_{\mathrm{f}} 5 \%$ superior a las placas fabricadas sin NS. Esta pequeña variación está asociada al efecto que tiene la NS en el tiempo de gel y la etapa de compactación [5] durante el proceso de infusión. Como se ha determinado anteriormente [24], el agregado de NS aumenta el tiempo de gel, indicando que habrá más tiempo para que la etapa de compactación del proceso de infusión permita obtener un espesor menor, aumentando ligeramente el $\mathrm{V}_{\mathrm{f}}[22]$. 
Los ensayos de tracción cuasiestáticos presentados en la Figura 4 mostraron un comportamiento que no es habitual en la literatura de materiales compuestos de matriz polimérica termoestable [22, 25, 26]. Como es sabido, para el caso de matrices termoestables, tanto de tipo poliéster como epoxi, la deformación a rotura no suele ser mayor al 5-6 \% [4]. Valores mayores son frecuentemente asociados a matrices elastoméricas o termoplásticas. Un comportamiento como el obtenido en los resultados presentados en la Figura 4 indica que la reorientación del refuerzo a medida que el material se deforma tendrá un rol importante en las propiedades mecánicas del material compuesto. Para bajas deformaciones, el refuerzo no cambiará substancialmente su orientación inicial de $+/-45^{\circ}$. Sin embargo, para deformaciones elevadas, el refuerzo se orientará en la dirección en donde se aplica la carga. Por ejemplo, si se considera una deformación hipotética del 10\%, esto implicará un cambio en la orientación del refuerzo en $5.32^{\circ}$. En otras palabras, el refuerzo estará inicialmente orientado en $+/-45^{\circ}$ pero, a medida que aumenta la deformación, se orientará hasta quedar en $+/-39.7^{\circ}$. Dado que el refuerzo es el principal contribuyente al módulo elástico y resistencia del material, se puede deducir que afectará sensiblemente sus propiedades mecánicas.

El módulo elástico de (E) para cada tipo de material se puede consultar en la Tabla 1. Como puede notarse, el E disminuyó con el agregado de NS, indicando que la nanopartícula afectó el grado de entrecruzamiento de la matriz. Teniendo en cuenta que la secuencia de laminado fue [+/-45] y asumiendo un estado uniaxial de tensión en la dirección $0^{\circ}$ y aplicando el criterio de Tsai-Hill para la falla de la primera lámina (FPF), tanto el módulo elástico como la resistencia son propiedades dominadas por la matriz [27] y, especialmente, del módulo de corte de la matriz $\left(\mathrm{G}_{12}\right)$. Esto indica que, para bajas deformaciones, el principal causante de cambios significativos en el E será el $\mathrm{G}_{12}$ de la matriz. Por ende, se puede deducir que el agregado de nanopartículas de sílice causó una disminución del $\mathrm{G}_{12}$ de la matriz [27]. Por otro lado, la resistencia última RUTS de las probetas ensayadas en este trabajo se puede consultar en Tabla 1. Como puede notarse, la RUTS disminuye en función del agregado de NS. En la literatura, asumiendo que ésta posee una dispersión nanométrica, es sabido que la NS posee un efecto contrario al observado en nuestro trabajo. Sin embargo, los resultados obtenidos en literatura no contemplan elevadas deformaciones. Para el caso estudiado en este trabajo, las elevadas deformaciones medidas, del orden del $10 \%$, indican que la contribución del refuerzo al cambio de la resistencia del material compuesto no es despreciable. Teniendo en cuenta los resultados cuasiestáticos a elevadas deformaciones (Figura 4), se puede deducir que la nanopartícula afectó la cinemática de desplazamiento del refuerzo, dado que los valores de resistencia dependieron también del contenido en peso de NS.

Los ensayos de ciclos a rotura (Nf) en función del \%RUTS se pueden observar en Figura 5. Como se mencionó anteriormente, para porcentajes de \%RUTS mayores al $60 \%$, los ciclos a rotura fueron reducidos. La explicación de este fenómeno está dada por la presencia de deformación inelástica que se acumula a medida que se acumulan ciclos. El nombre de este fenómeno es "ratchetting" y está fundamentalmente asociado al comportamiento viscoelástico de la matriz poliuretano [4]. Como los ciclos fueron ejecutados a una frecuencia de $2 \mathrm{~Hz}$, el tiempo entre cada ciclo no permitió una relajación de tensiones. Está hipótesis se demuestra en la Figura 7, en donde puede observarse que a una frecuencia de 2HZ, el desplazamiento del cabezal de la máquina de ensayos dinámicos aumentó significativamente en función de la cantidad de ciclos. Tal efecto no se observa en ensayos similares de matrices termoestables [6, 8, 9, 17, 18, 28, 29]. Esto indica que los resultados están afectados por un acentuado comportamiento viscoelástico. Por ende, esto generó un constante aumento de la posición del cabezal, llegando a valores muy superiores a la deformación de rotura de la matriz poliuretano. Dentro de este régimen (> 60 \%RUTS), la incorporación de NS en la matriz provocó una disminución de la performance frente a la fatiga. Para el caso de tensiones por debajo del $60 \%$ RUTS, los ciclos a rotura aumentaron significativamente a valores habitualmente encontrados en literatura. Asimismo, como puede deducirse de las predicciones del modelo empírico (Figura 5), el agregado de NS permitió mejorar la performance a la fatiga. Si se tiene en cuenta que la aplicación de estos materiales compuestos radica en su uso en aspas eólicas, es coherente que las tensiones de diseño sean bastante inferiores al $60 \%$ RUTS, dado que los coeficientes de seguridad implican que los diseños deben trabajar en zonas alejadas de la resistencia última. Teniendo en cuenta esto, se puede deducir que el agregado de NS en la matriz aumentará la performance a la fatiga de las zonas donde se utilice el material compuesto nanoestructurado.

Para corroborar estos resultados, se decidió estudiar el módulo elástico (E) residual en función de la cantidad de ciclos a una tensión del 60\% UTS. Como puede notarse en la Figura 6, el agregado de NS generó una mejoría en la performance del material compuesto, aumentando los ciclos a rotura en un promedio del $18.8 \%$.

Finalmente, se procedió a realizar ensayos de probetas manteniendo las variables del ensayo de fatiga y disminuyendo la frecuencia (Figura 7). Como puede notarse, la disminución de la frecuencia generó una sensible disminución del efecto viscoelástico (del inglés "ratchetting"), disminuyendo significativamente la posición del cabezal en función de la cantidad de ciclos así como la deformación residual medida por inspección visual de la probeta fallada (Figura 7). Es importante destacar que la evolución de temperatura asociada al cambio de frecuencia del ciclado de fatiga es un aspecto fundamental para analizar el comportamiento viscoelástico. Los autores se encuentran realizando experimentos con mediciones in-situ de temperatura para cuantificar este fenómeno.

Comparando los resultados de las curvas S-N de materiales compuestos de similares características elaborados por 
Dyer et al. [30] se puede deducir que la performance a la fatiga y el comportamiento estático es muy superior usando matrices nanoestructuradas. Por ejemplo, bajo ciclos de tensión máxima a $30 \mathrm{MPa}$, Dyer [30] obtuvo ciclos a la rotura del orden de $10^{3}$, valores muy por debajo de los obtenidos en este trabajo. Otros trabajos también confirman esta tendencia [2, 6-8].

\section{CONCLUSIONES}

Se fabricaron placas de material compuesto con refuerzo bidireccional $[+/-45]_{4 \mathrm{~S}}$ y matrices poliuretano nanoestructuradas con nanosílice hasta el $2 \%$ en peso usando la técnica de infusión asistida por vacío. A pesar del aumento de viscosidad causado por la NS, se lograron elevados contenidos de refuerzo $\left(\mathrm{V}_{\mathrm{f}}\right)$ y una buena impregnación. Se comprobó la factibilidad del uso de matrices nanoestructuras en el proceso de IAV. A pesar del aumento de viscosidad provocado por la NS, se puede lograr una impregnación y consolidación adecuada del refuerzo sin cambios significativos de la geometría de la zona de infusión.

Los ensayos cuasiestáticos presentaron una elevada deformación a rotura, indicando que la matriz le proveía al material compuesto una elevada deformación y, en consecuencia de esto, la contribución del refuerzo a la resistencia del material y su módulo fue más significativa que la ocasionada por la nanosílice. Los ensayos a fatiga presentaron un comportamiento dual, en donde para tensiones por debajo del $60 \%$ de la RUTS se observaron elevados ciclos a fatiga así como una mejoría en la performance a fatiga para el caso de materiales compuestos nanoestructurados. Estos resultados fueron confirmados por mediciones del módulo residual realizadas al $60 \%$ del RUTS, confirmando el efecto benéfico de la nanosílice. Sin embargo, para tensiones por arriba del $60 \%$ de la RUTS, se observaron muy bajos ciclos a rotura. La causa de esto fue el elevado comportamiento viscoelástico de la matriz poliuretano ("ratchetting"), que no permitió que ésta se relaje a medida que avanzaban los ciclos de fatiga. Esto dió lugar a una elevada acumulación de energía inelástica que derivó en fallas prematuras. Los resultados de este trabajo indican que el material propuesto es un excelente candidato para materiales compuestos aplicados en palas eólicas, dado que permite disminuir la acumulación del daño y mejorar la resistencia a la fatiga.

\section{AGRADECIMENTOS}

Se agradece a Matías Nonna y Matías Ferreyra (Huntsman Argentina) por proveer la matriz poliuretano. Este proyecto se realizó usando fondos del proyecto PICT 2015-N0475 expedido por la Agencia Nacional de Promoción Científica Argentina.

\section{BIBLIOGRAFIA}

[1] MALLICK, P.K., 2.09 - Particulate and Short Fiber Reinforced Polymer Composites, in Comprehensive Composite Materials, K. Editors-in-Chief: Anthony and Z. Carl, Editors. 2000, Pergamon: Oxford. pp. 291-331.

[2] HARRIS, B., Fatigue in composites: science and technology of the fatigue response of fibre-reinforced plastics. Woodhead Publishing, 2003.

[3] YANG, J., et al., Structural investigation of composite wind turbine blade considering structural collapse in full-scale static tests. Composite Structures. n. 97, pp. 15-29, 2013.

[4] SZYCHER M., Szycher handbook of Polyurethanes. 2013, Florida, NY: Taylor \& Francis group LLC.

[5] HAMIDI, Y.K. AND M.C. ALTAN, Process induced defects in liquid molding processes of composites. International Polymer Processing, v. 32, n. 5, pp. 527-544, 2017.

[6] LOOS, M., et al., Enhancement of fatigue life of polyurethane composites containing carbon nanotubes. Composites Part B: Engineering, v. 44, n. 1, pp. 740-744, 2013.

[7]AGARWAL, B., J.W. DALLY, Prediction of low-cycle fatigue behaviour of GFRP: an experimental approach. Journal of Materials Science, v. 10, n. 2, pp. 193-199, 1975.

[8] QIAO, P. AND M. YANG, Fatigue life prediction of pultruded E-glass/polyurethane composites. Journal of Composite Materials, v. 40, n.9, pp. 815-837, 2006.

[9] KHAN, S.U., et al., Fatigue damage behaviors of carbon fiber-reinforced epoxy composites containing nanoclay. Composites Science and Technology, v. 70, n. 14, pp. 2077-2085, 2010.

[10] CHIACCHIARELLI, L., et al., The relationship between nanosilica dispersion degree and the tensile properties of polyurethane nanocomposites. Colloid and Polymer Science, p. 1-9, 2013. 
[11] CHIACCHIARELLI, L.M., et al., A polycaprolactone-based compatibilization treatment to improve dispersion and interphase structure of silica polyurethane composites. Polymer Engineering \& Science, v. 54, n. 8, op. 1817-1826, 2014.

[12] KENNY, J.M., L. TORRE, L.M. CHIACCHIARELLI, The effect of processing routes on the thermal and mechanical properties of poly (urethane-isocyanurate) nanocomposites. Journal of Applied Polymer Science, v. 132, n. 45, 2015.

[13] MAZUMDAR, S., Composites manufacturing: materials, product, and process engineering. 2001: CRC press.

[14] CHIACCHIARELLI L. M., TORRE L., AND K.J. M., Introducing nanostructured polyurethanes in high volume applications. SAMPE Europe SEICO Conference 2014.

[15] CHIACCHIARELLI, L.M., R. PETRUCCI, L. TORRE, Enhanced fracture toughness of nanostructured carbon fiber reinforced poly (urethane isocyanurate) composites at low concentrations. Polymer Engineering \& Science, 2017.

[16] POTTER, K., Resin transfer moulding. Springer Science \& Business Media, 1997.

[17] KIM, J.-H., et al., Review of nanocellulose for sustainable future materials. International Journal of Precision Engineering and Manufacturing-Green Technology, v. 2, n. 2, pp. 197-213, 2015.

[18] CHIRAYIL, C.J., L. MATHEW, S. THOMAS, REVIEW OF RECENT RESEARCH IN NANO CELLULOSE PREPARATION FROM DIFFERENT LIGNOCELLULOSIC FIBERS. Reviews on advanced materials science, n. 37, 2014.

[19] CHIACCHIARELLI L. M., et al., The relationship between nanosilica dispersion degree and the tensile properties of polyurethane nanocomposites. Submitted to Colloid and Polymer Science (Minor Revisions).

[20] BROWN M.W.R. , COATES P.D., JOHNSON A.F., Reactive Processing of Polymers. Rapra Technologies Ltd., v. 7, n. $1,1994$.

[21] KELLER, A., et al., Fast-curing epoxy polymers with silica nanoparticles: properties and rheo-kinetic modelling. Journal of Materials Science, v. 51, n. 1, pp. 236-251, 2016.

[22] FORESTI, M., P. CERRUTTI, A. VAZQUEZ, Bacterial nanocellulose: Synthesis, properties and applications. Polymer Nanocomposites Based on Inorganic and Organic Nanomaterials; John Wiley \& Sons, Inc.: Hoboken, NJ, USA, pp. 39-61, 2015.

[23] MALLICK, P.K., Fiber-reinforced composites: materials, manufacturing, and design. CRC press, 2007.

[24] CHIACCHIARELLI, L.M., et al., Cure kinetics of a highly reactive silica-polyurethane nanocomposite. Thermochimica Acta, v. 549, n. 0, pp. 172-178, 2012.

[25] HODGKINSON, J.M., Mechanical testing of advanced fibre composites. 2000, Abington: Woodhead Publishing Limited.

[26] HINCKLEY, W.M. and J. Yang, Analysis of rigid polyurethane foam as a shock mitigator. Experimental Mechanics, v. 15, n. 5 , pp. 177-183, 1975.

[27] DANIEL, I.M., et al., Engineering mechanics of composite materials. Vol. 3. 1994: Oxford university press New York.

[28] CHARREAU, H., M. L FORESTI, A. VÁZQUEZ, Nanocellulose patents trends: a comprehensive review on patents on cellulose nanocrystals, microfibrillated and bacterial cellulose. Recent patents on nanotechnology, v. 7, n. 1, pp. 56-80, 2013.

[29] LU, G., T. YU, Energy absorption of structures and materials. Elsevier, 2003.

[30] DYER, K., D. ISAAC, Fatigue behaviour of continuous glass fibre reinforced composites. Composites Part B: Engineering, v. 29, n. 6, pp. 725-733, 1998.

\section{ORCID}

Nicolas Oyarzabal Alberto Belardi

Leonel M Chiacchiarelli
https://orcid.org/0000-0002-2083-178X

https://orcid.org/0000-0003-0286-282X

https://orcid.org/0000-0002-7342-1784 